\title{
AGN and starburst radio activity in the A3558 cluster complex ${ }^{\star}$
}

\author{
S. Giacintucci ${ }^{1}$, T. Venturi ${ }^{2}$, S. Bardelli ${ }^{1}$, D. Dallacasa ${ }^{2,3}$, and E. Zucca ${ }^{1}$ \\ 1 INAF - Osservatorio Astronomico di Bologna, via Ranzani 1, 40127 Bologna, Italy \\ 2 Istituto di Radioastronomia del CNR, via Gobetti 101, 40129, Bologna, Italy \\ 3 Dipartimento di Astronomia, Università di Bologna, via Ranzani 1, 40127, Bologna, Italy
}

Received 10 June 2003 / Accepted 2 February 2004

\begin{abstract}
We present Very Large Array (VLA) $1.4 \mathrm{GHz}(21 \mathrm{~cm})$ observations of the region between the centres of A3558 and A3562, in the major cluster merger complex of the Shapley Concentration. Our final catalogue includes a total of 174 radio sources above the flux density limit of $0.25 \mathrm{mJy} \mathrm{b}^{-1}$. By cross-correlation with optical and spectroscopic catalogues we found 33 optical counterparts belonging to the Shapley Concentration.

We investigated the effects of cluster merger on the radio emission properties of the galaxy population by means of the radio source counts and the radio luminosity functions (RLF). We found that the radio source counts are consistent with the field source counts. The RLF of elliptical and S0 galaxies in the region surveyed, is consistent with the "universal" RLF for early-type galaxies. This result suggests that the deficit in radio galaxies found in our previous work over the whole A3558 chain is entirely due to the cluster A3558. A population of faint radio galaxies $\left.\left(\log P_{1.4 \mathrm{GHz}}(\mathrm{W} \mathrm{Hz})^{-1}\right) \lesssim 22\right)$ is also found. Half of these objects are also blue, suggesting that starburst is the main mechanism driving the radio emission. Finally, we detected 14 spiral radio galaxies, whose ratio between radio and optical emission is similar to those found in galaxies located in rich and dynamically evolved clusters.

Our results are briefly discussed in the light of the age and stage of the merger in the A3558 cluster complex.
\end{abstract}

Key words. radio continuum: galaxies - galaxies: clusters: general - galaxies: clusters: individual: A3558, A3562, SC 1329-313, SC 1327-312

\section{Introduction}

Evidence is accumulating that the interaction processes between clusters of galaxies, known as cluster mergers, may significantly affect the radio emission characteristics of the cluster galaxy population. In particular, the evolution of galaxies and the properties of their nuclear and/or star forming activity are undoubtely influenced by interaction with the environment. Cluster merging and group accretion seem to play an important role, but it is not yet completely understood how the merging environment affects the nuclear and starburst emission in galaxies. Owen et al. (1999) suggested that merging may trigger radio emission, both in the form of nuclear activity and starburst phenomena. Burns et al. (1994) interpreted the presence of post-starburst galaxies in the X-ray and radio bridge connecting the Coma cluster and the NGC 4839 group as the consequence of a recent merging process between these two structures. Using numerical simulations, Bekki (1999) concluded that the tidal gravitational field of a cluster merger may drive a

Send offprint requests to: T. Venturi, e-mail: tventuri@ira.cnr.it

* Full Tables 3 and 4 are only available in electronic form at the CDS via anonymous ftp to

cdsarc.u-strasbg.fr $(130.79 .128 .5)$ or via

http://cdsweb.u-strasbg.fr/cgi-bin/qcat?J/A+A/419/71 considerable transfer of gas to the central regions of galaxies, leading to enhanced star formation activity or feeding of the central engine of active galactic nuclei. Vollmer et al. (2001) suggested that a local burst of star formation could be due to re-accretion of gas after a ram pressure stripping event, which is thought to be responsible for the HI deficit of spiral galaxies in the central regions of nearby clusters with respect to the field galaxies of the same morphological type and optical size (Gunn \& Gott 1972; Bothun \& Dressler 1986). On the other hand, Fujita et al. (1999) and Balogh et al. (1998) claimed that gas stripping resulting from ram pressure is important in preventing gas supply to the central regions of the galaxies and may suppress star formation. Finally Venturi et al. (2000, hereinafter V2000) found evidence that merging may anticorrelate with the radio emission, possibly switching off existing radio galaxies, or temporarily inhibiting the nuclear radio activity.

A major problem in our current knowledge of the role of cluster mergers in the radio emission and in the interpretation of the observations is the lack of statistics. Only few clusters of galaxies have been deeply imaged over their whole extent (i.e. A2125, Dwarakanath \& Owen 1999; A2255, Miller \& Owen 2003, hereinafter MO03; A2256, Miller et al. 2003; A2645, Owen et al. 1999; the Shapley supercluster chains A3528, A3558 and A3571, Venturi et al. 2000, 2001 and 2002 


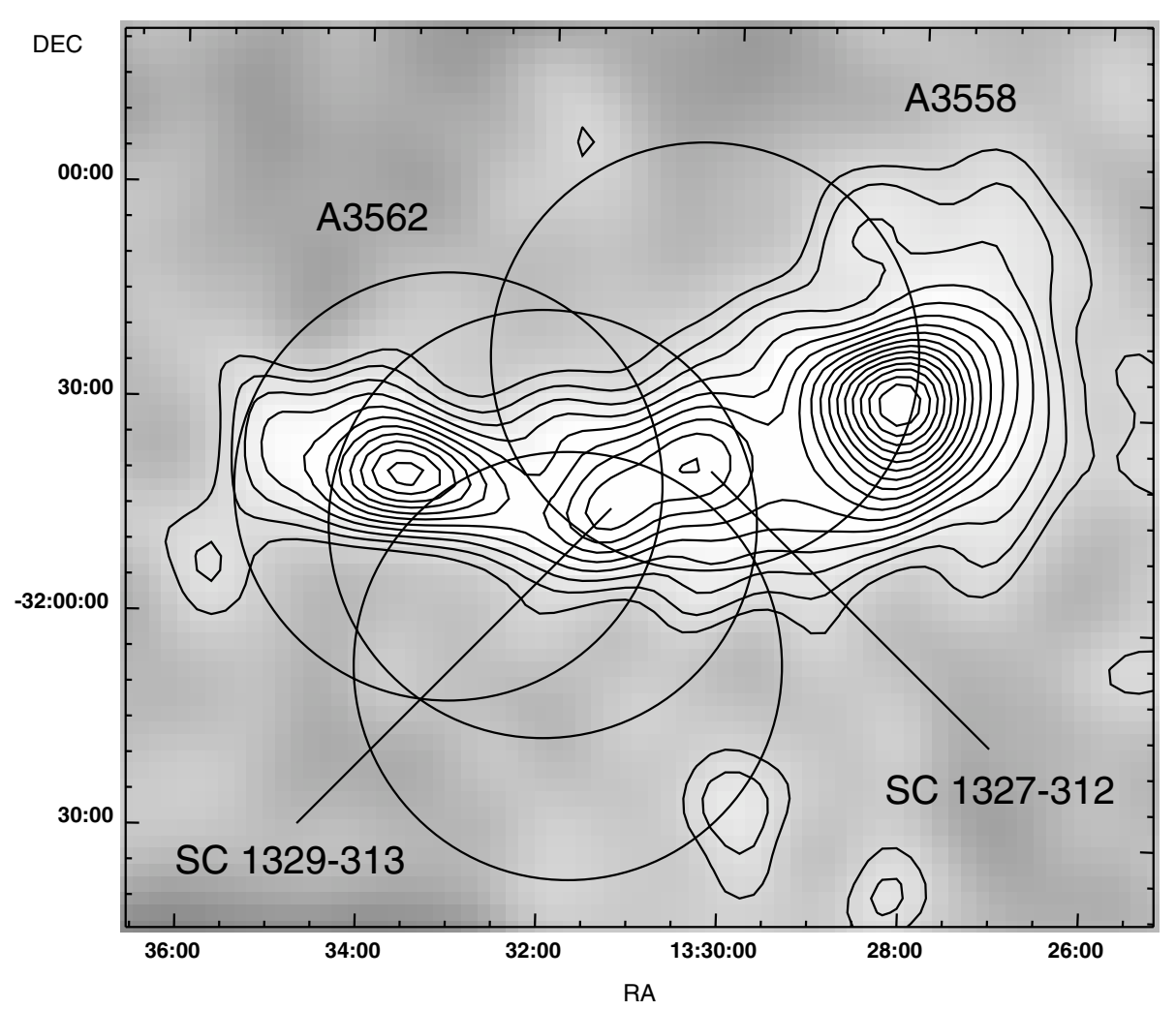

Fig. 1. Grey scale and isocontours of the galaxy density to $b_{\mathrm{J}}=19.5$ in A3558-C from the COSMOS catalogue. The cluster A3556, located westward of A3558, is not included in the figure. The centres of the superposed circles correspond to the pointing centres of the observations. The radius of each circle is 30 arcmin (i.e. primary beam size of the VLA at $1.4 \mathrm{GHz}$ ).

respectively), and the complexity of the phenomenon clearly needs more observational support. Cluster mergers evolve over a timescale of $\sim \mathrm{Gyr}$, while typical ages of radio sources are of the order of few times $10^{7}-10^{8}$ years, therefore it is of crucial importance to identify the effects of the various stages of a merger event. For this reason it is essential to carry out deep radio observations of a large number of galaxy clusters, at different merger stages.

To study the effects of a major cluster merger event after the first core-core encounter on the nuclear and starburst radio emission, in this paper we present $1.4 \mathrm{GHz}$ Very Large Array (VLA) observations of the cluster A3562 and the poor groups SC 1329-313 and SC 1327-312, at the eastern side of the A3558 cluster complex. In the following we will refer to the area surveyed as A3558-C. This work is part of a larger project which aims to study the influence of the ongoing merger in the core of the Shapley Concentration on the radio/optical properties of the cluster galaxies, both from a statistical point of view and through a detailed analysis of the physical properties of the extended radio galaxies. In Sect. 2 we briefly overview the optical and X-ray properties of A3558-C, with emphasis on the cluster A3562 and on the poor groups SC 1329-313 and SC 1327-312; the observations and data reduction are presented in Sect. 3; in Sect. 4 we present the $1.4 \mathrm{GHz}$ radio sample and the source counts; in Sect. 5 we deal with the optical identifications and in Sect. 6 we present the general properties of the radio galaxies in this region; the results of the analysis of the radio luminosity function (RLF) for the early-type and late-type galaxy populations are given in Sect. 7; discussion and conclusions are given in Sect. 8.

We assume $H_{0}=100 \mathrm{~km} \mathrm{~s}^{-1} \mathrm{Mpc}^{-1}$ and $q_{0}=0.5$. If we define $h=H_{0} / 100$, at the average redshift of the Shapley Concentration $(z=0.05)$ this leads to a linear scale of $1 \operatorname{arcsec}=0.67 h^{-1} \mathrm{kpc}$. We will assume $S \propto v^{-\alpha}$.

\section{The cluster merger in A3558-C}

The dynamical centre of the Shapley Supercluster can be identified as the A3558 cluster complex, a chain of interacting clusters located at a mean redshift $z=0.0483$ and spanning $\sim 7.5 h^{-1} \mathrm{Mpc}$ (projected), almost orthogonal to the line of sight. The chain is formed by the three ACO (Abell et al. 1989) clusters A3558, A3562 and A3556, and includes the two poor groups SC 1327-312 and SC 1329-313 (Fig. 1).

The A3358 cluster complex has been extensively studied over a wide range of bands, from radio wavelengths up to X-ray energies, and the physical connection and the merging stage of all clusters in this region is suggested by observational data in the radio (V2000, Venturi et al. 2003, hereinafter V2003), optical (Bardelli et al. 1994) and X-ray bands (Bardelli et al. 1996; Ettori et al. 1997).

The distribution of the hot gas in this region remarkably follows the distribution of the optical galaxies, and a detailed substructure analysis carried out by Bardelli et al. (1998a) revealed that the whole structure is characterized by a large number of subcondensations, further evidence of its dynamical activity. In addition, the galaxy distribution in the A3558 chain 
Table 1. Cluster properties.

\begin{tabular}{|c|c|c|c|c|c|c|c|c|}
\hline Cluster & $\begin{array}{c}\mathrm{RA}_{\mathrm{J} 2000} \\
(2)\end{array}$ & $\begin{array}{c}\operatorname{Dec}_{J 2000} \\
\text { (3) }\end{array}$ & $\begin{array}{c}B-M \\
\text { (4) }\end{array}$ & $R$ & $\begin{array}{c}\langle v\rangle \\
\mathrm{km} \mathrm{s}^{-1} \\
(6)\end{array}$ & $\begin{array}{c}\sigma_{\mathrm{v}} \\
\mathrm{km} \mathrm{s}^{-1} \\
(7)\end{array}$ & $\begin{array}{c}k T \\
\mathrm{keV} \\
(8)\end{array}$ & $\begin{array}{c}L_{\mathrm{X}}(2-10 \mathrm{keV}) \\
h^{-2} \mathrm{erg} \mathrm{s}^{-1} \\
(9)\end{array}$ \\
\hline SC 1327-312 & 132947 & -313629 & - & - & $14844_{-211}^{+105 a}$ & $691_{-146}^{+158 a}$ & $4.11_{-0.36}^{+0.43 c}$ & $3.76 \times 10^{43 c}$ \\
\hline SC $1329-313$ & 133136 & -314846 & - & - & $\begin{array}{l}\text { (T520) } 13280_{-95}^{+80 b} \\
\text { (T496) } 14690_{-99}^{-101 b}\end{array}$ & $\begin{array}{l}482_{-49}^{+87 b} \\
537_{-32}^{+87 b}\end{array}$ & $3.49_{-0.24}^{+0.27 c}$ & $2.36 \times 10^{43 c}$ \\
\hline A 3562 & 133330 & -314000 & I & 2 & $14492_{-286}^{+225 a}$ & $913_{-96}^{189 a}$ & $5.13_{-0.19}^{+0.21 d}$ & $5.25 \times 10^{43 d}$ \\
\hline
\end{tabular}

Notes to Table 1. The coordinates of the centre for A3562 are taken from Abell et al. (1989), the coordinates for SC 1327-312 are from Bardelli et al. (1996) and for SC 1329-313 from Breen et al. (1994). Note that the SC 1329 group has a bimodal velocity distribution: the optical clumps associated to the two velocity peaks are named T520 and T496 (Bardelli et al. 1998a). ${ }^{a}$ Bardelli et al. (1998b), ${ }^{b}$ Bardelli et al. (1998a), ${ }^{c}$ Bardelli et al. (2002), ${ }^{d}$ Ettori et al. (2000).

Table 2. Logs of the Observations.

\begin{tabular}{cccccc}
\hline \hline $\begin{array}{c}\text { Field } \\
\#\end{array}$ & RA $_{\mathrm{J} 2000}$ & Dec $_{\mathrm{J} 2000}$ & Array & $\begin{array}{c}\text { Int. time } \\
(\mathrm{h})\end{array}$ & $\begin{array}{c}\mathrm{rms}(1.4 \mathrm{GHz}) \\
\mathrm{mJy} / \mathrm{b} \\
(1)\end{array}$ \\
$(2)$ & $(3)$ & $(4)$ & $(5)$ & $(6)$ \\
\hline 1 & 133332 & -314060 & DnC & 1 & 0.05 \\
2 & 133202 & -314660 & DnC & 1 & 0.05 \\
3 & 133019 & -312257 & DnC & 1 & 0.05 \\
4 & 133143 & -320645 & DnC & 1 & 0.05 \\
\hline
\end{tabular}

resembles the results of the numerical simulations carried out by Roettiger et al. (1997) for the case of a collision of two clusters. The observational scenario suggests that the whole region is the result of a major merger with the cluster A3558, seen after the first core-core encounter (Bardelli et al. 1998b, 2002). All the structure visible in the chain beyond A3558 itself, i.e. A3556, A3562 and the two small groups SC 1327-312 and SC 1329-313, would be the remains of the colliding cluster (see Fig. 1). In the region between A3558 and A3562, where the initial position of the merger shock is expected, Bardelli et al. (1998b) found an enhanced fraction of blue galaxies.

The whole A3558 cluster complex was surveyed at 22/13 cm with the Australia Telescope Compact Array (ATCA, Venturi et al. 1997; Venturi et al. 1998, V2000) and a number of interesting results emerged from the radio analysis. In particular, the outskirts of the chain, i.e. A3556 and A3562, contain the largest number of cluster radio sources and the four extended radio galaxies. Moreover, the radio luminosity function computed for elliptical galaxies over the whole complex is considerably lower that the "universal" radio luminosity function for ellipticals derived by Ledlow \& Owen (1996, hereinafter LO96), suggesting a possible connection with the cluster merger in this region. Finally, a cluster radio halo was detected at the centre of A3562 (V2000, V2003), as further evidence of the role of merger on the cluster radio emission.

The properties of A3562, SC 1327-312 and SC 1329-313 are summarised in Table 1, where we report:

- Columns 2 and 3: J2000 coordinates;

- Columns 4 and 5: Bautz-Morgan type and richness;
- Columns 6 and 7: mean heliocentric velocity and velocity dispersion;

- Columns 8 and 9: X-ray temperature and luminosity.

\section{Observations and data reduction}

The $1.4 \mathrm{GHz}(21 \mathrm{~cm})$ observations of A3562 and of the two SC groups 1327-312 and 1329-313 were carried out on 10 July 2000 with the Very Large Array (VLA), operating in the DnC configuration. The observations were carried out in continuum mode with a $50 \mathrm{MHz}$ bandwidth, for a total duration of $4 \mathrm{~h}$. We switched among the four fields every $4.5 \mathrm{~min}$. 3C 286 was used as primary calibrator, and $1316-336$ as phase calibrator. The logs of the observations are given in Table 2 .

The region of interest was covered with four different pointings; the resolution is $41.98^{\prime \prime} \times 35.13^{\prime \prime}$ at the declination of the A3558 complex.

The observations were carried out using the mosaicing facility of the VLA. Pointing \# 1 and \# 2 were chosen to be centered respectively on the head-tail J1333-3141 in the centre of A3562, where the radio halo lies, and on the diffuse radio source J1332-3146a in the region between A3562 and the SC 1329-313 group (V2000). The remaining pointings were chosen to remove the sidelobes from the confusing sources at the field edges and to ensure uniform sensitivity over the whole region between the centre of A3562 and the SC groups.

The data reduction was carried out using the standard procedure (calibration, Fourier inversion, clean and restore) of the NRAO AIPS (Astronomical Image Processing System) package. We reduced the data and imaged each field separately. 
The final images were then mosaiced (i.e. linearly combined) using the AIPS task LTESS. The average noise in the individual final images is $\sim 0.05 \mathrm{mJy}^{-1}$ in all four fields. We assume as reliable all detections with flux density peak $S_{1.4 \mathrm{GHz}} \geq$ $0.25 \mathrm{mJy} \mathrm{b}^{-1}$, corresponding to a $5 \sigma$ confidence level. Such a limit implies a limit on the radio power of $\log P_{1.4 \mathrm{GHz}}$ $\left(\mathrm{W} \mathrm{Hz}{ }^{-1}\right)=20.83$ at the average distance of A3558-C. We note that our detection limit is near the confusion limit at the frequency and resolution of the images presented here. To evaluate this effect, we cross-checked our detections on a pure uniform weight image, where the confusion is considerably lower, and considered as reliable detections only those sources passing the cross-check.

\section{The sample of radio sources}

We detected a total of 174 radio sources at $1.4 \mathrm{GHz}$ above the peak flux density limit of $0.25 \mathrm{mJy} \mathrm{b}^{-1}$. The source list is reported in Table 3, where we give:

- Columns 1-3: name (VLA-) and J2000 position;

- Column 4: flux density at $1.4 \mathrm{GHz}$ corrected for the primary beam attenuation. The values were derived with task JMFIT for unresolved or marginally resolved sources, while they were obtained by means of TVSTAT for the extended sources;

- Column 5: radio morphology. We classified the sources as unres. $=$ unresolved and ext. $=$ extended. Moreover we note that $\mathrm{D}=$ double and HT = head-tail. For the double source we give the position of the radio barycentre and for the extended sources we give the position of the radio peak.

As it is clear from Table 3 most of the radio sources detected at $1.4 \mathrm{GHz}$ are unresolved; only 23 radio sources are extended, i.e. $13 \%$ of the total.

Our observations confirm the presence of a radio halo at the centre of A3562 (not listed in Table 3), encompassing the head-tail radio galaxy J1333-3141. The halo has a flux density of $S_{1.4 \mathrm{GHz}}=20 \mathrm{mJy}$, a low surface brightness $\left(\leq 0.175 \mathrm{mJy} \mathrm{b}^{-1}\right)$ and an irregular shape, with largest angular size of $\sim 8^{\prime}$. The properties and the origin of this source are discussed in a different paper (V2003).

We note that the region presented in this paper (A3558-C) partly overlaps with the area covered in the ATCA survey presented in V2000. We carefully checked all sources in the common region, and found that all differences can be accounted for either by the different $u-v$ coverage, resolution and/or sensitivity of the two sets of observations. Due to the different resolutions $\left(\sim 10^{\prime \prime} \times 5^{\prime \prime}\right.$ for the ATCA observations), in a number of cases the radio emission detected with the present observations is actually a blend of two or more sources in the ATCA $22 \mathrm{~cm}$ sample. Those cases of source blending relevant to the discussion of this paper are briefly commented in the Appendix.

\subsection{Radio source counts}

We computed the source counts for our radio sample in A3558-C, to test whether the optical galaxy overdensity and the ongoing merging scenario proposed here reflect a higher number of radio sources with respect to the field radio sources counts.

Since the sensitivity in the final images of the four fields covered by our observations is not uniform due to the VLA primary beam attenuation, the $1.4 \mathrm{GHz}$ sample is not complete to the flux density limit of $0.25 \mathrm{mJy}$. For this analysis we therefore considered only the radio sources with $S_{1.4 \mathrm{GHz}} \geq 0.50 \mathrm{mJy}$ within a radius of 15.5 arcmin from the centre of each field. At such a distance the primary beam attenuation of the VLA at $1.4 \mathrm{GHz}$ is reduced by a factor of two, and sources with flux density $S_{1.4 \mathrm{GHz}} \geq 0.50 \mathrm{mJy}$ are seen as sources with $S_{1.4 \mathrm{GHz}} \geq$ $0.25 \mathrm{mJy}$ before the correction. At the distance of A3558-C this limit implies a radio power $\log P_{1.4 \mathrm{GHz}}\left(\mathrm{W} \mathrm{Hz}^{-1}\right)=21.13$. We counted 92 radio sources with $S \geq 0.5 \mathrm{mJy}$ in an area of $0.75 \mathrm{deg}^{-2}$.

We compared our results with the radio source field counts in Prandoni et al. (2001), who counted 1752 radio sources with a flux density at $22 \mathrm{~cm} S_{22} \geq 1 \mathrm{mJy}$ over an area of $25.82 \mathrm{deg}^{-2}$. Taking into account the completeness flux density limit of Prandoni et al. (2001) and the different area covered in their survey, we counted 88 radio sources, to be compared to the number expected from the background counts, i.e. 72.3. These two numbers differ by $\sim 20 \%$, and the difference mainly comes from the two flux density bins in the range 13.12-26.4 mJy, where we found 12 radio sources, against the expected 4 . This excess is significant at a $2 \sigma$ level. We note that only 2 out of the 12 radio sources belong to the Shapley Concentration. If we exclude these two bins from our analysis, we find 76 radio sources, while the counts from the background give 68.2, therefore there is still a marginal excess in our data of $\sim 10 \%$.

\section{Optical identifications}

In order to find as many optical counterparts as possible, we cross-correlated the radio positions of all sources in our sample with four different optical catalogues. Our reference catalogue is the COSMOS/UKST Southern Sky Object Catalogue (Yentis et al. 1992), limited to $b_{\mathrm{J}}=19.5$; for fainter magnitudes we considered also the Super COSMOS/USKT Southern Sky Object Catalogue (Hambly et al. 2001), the APM Catalogue (Maddox et al. 1990) and the MGP catalogue (Metcalfe et al. 1994).

These catalogues have a claimed positional accuracy of $\sim 0.25^{\prime \prime}$, but given the errors that could be introduced by transforming the sky image on the plate frame, we adopted a mean optical positional uncertainty of 1.5 arcsec (Unewisse et al. 1993).

The radio positional error depends on the beam size and on the source flux density (Prandoni et al. 2000). With the parameters of our observations we estimate an average position uncertainty of 2.5 arcsec both in right ascension and declination. For the faintest sources in the sample $(5 \sigma)$ the positional error rises to $\sim 4.2^{\prime \prime} \times 3.5^{\prime \prime}$ along the beam axis $(\mathrm{HPBW} /(2 \times$ SNR), SNR being the signal-to-noise ratio).

To make sure that no identification was missed, we examined the photometric catalogue of optical galaxies in this region in Metcalfe et al. (1994) and we overplotted all the radio sources in our sample on the optical red Digitized Sky Survey DSS-2 images and carried out a careful visual inspection. 
Table 3. Source list and flux density values.

\begin{tabular}{|c|c|c|c|c|}
\hline $\begin{array}{l}\text { Name } \\
\text { VLA- }\end{array}$ & $\mathrm{RA}_{\mathrm{J} 2000}$ & $\overline{\operatorname{Dec}_{\mathrm{J} 2000}}$ & $\begin{array}{c}S_{1.4 \mathrm{GHz}} \\
(\mathrm{mJy})\end{array}$ & Radio morphology \\
\hline J1328-3119 & 132829.35 & -311914.7 & 66.34 & unres. \\
\hline J1328-3134 & 132831.50 & -313450.4 & 126.80 & unres. \\
\hline $\mathrm{J} 1328-3115$ & 132835.09 & $-31 \quad 1522.1$ & 1.97 & unres. \\
\hline J1329-3126 & 132900.02 & -312626.0 & 81.92 & ext. \\
\hline J1329-3131 & 132904.61 & -313102.1 & 86.80 & unres. \\
\hline J1329-3112 & 132909.12 & -311256.8 & 5.37 & unres. \\
\hline J1329-3121 & 132913.32 & -312151.1 & 23.65 & unres. \\
\hline J1329-3129a & 132929.43 & -312943.7 & 18.90 & unres. \\
\hline $\mathrm{J} 1329-3100$ & 132931.67 & -310017.0 & 5.48 & unres. \\
\hline J1329-3116 & 132931.88 & -311651.9 & 25.06 & ext. \\
\hline J1329-3102 & 132939.15 & -310208.3 & 2.15 & unres. \\
\hline J1329-3119a & 132949.32 & -311937.3 & 6.02 & unres. \\
\hline J1329-3101 & 132949.42 & -310144.6 & 3.03 & unres. \\
\hline J1329-3122 & 132950.76 & -312255.6 & 22.43 & ext. \\
\hline J1329-3057 & 132951.22 & -305747.2 & 65.38 & unres. \\
\hline J1329-3056 & 132952.90 & -305600.7 & 123.04 & ext. (D) \\
\hline $\mathrm{J} 1329-3119 \mathrm{~b}$ & 132955.02 & -311957.7 & 4.53 & unres. \\
\hline$J 1329-3129 b$ & 132955.32 & -312936.7 & 8.62 & unres. \\
\hline $\mathrm{J} 1330-3124 \mathrm{a}$ & 133005.10 & -312436.3 & 6.89 & unres. \\
\hline $\mathrm{J} 1330-3144 \mathrm{a}$ & 133005.20 & -314451.7 & 4.01 & unres. \\
\hline $\mathrm{J} 1330-3113$ & 133005.59 & $-31 \quad 1344.8$ & 1.79 & unres. \\
\hline $\mathrm{J} 1330-3215 \mathrm{a}$ & 133005.60 & -321554.3 & 1.81 & unres. \\
\hline $\mathrm{J} 1330-3143 \mathrm{a}$ & 133005.82 & -314342.2 & 4.02 & unres. \\
\hline $\mathrm{J} 1330-3102$ & 133007.17 & -310219.4 & 10.07 & ext. \\
\hline J1330-3103 & 133008.55 & -310346.2 & 1.08 & unres. \\
\hline $\mathrm{J} 1330-3144 \mathrm{~b}$ & 133008.56 & -314402.8 & 3.26 & unres. \\
\hline $\mathrm{J} 1330-3116$ & 133009.79 & $-31 \quad 1610.8$ & 2.68 & unres. \\
\hline $\mathrm{J} 1330-3124 \mathrm{~b}$ & 133010.06 & -312405.6 & 5.81 & unres. \\
\hline $\mathrm{J} 1330-3214$ & 133014.71 & -321446.3 & 1.94 & unres. \\
\hline J1330-3122 & 133019.06 & -312258.2 & 439.08 & unres. \\
\hline J1330-3159 & 133019.41 & -315940.0 & 2.05 & unres. \\
\hline J1330-3201 & 133021.69 & -320133.3 & 3.27 & unres. \\
\hline J1330-3129 & 133030.79 & -312959.3 & 14.13 & unres. \\
\hline $\mathrm{J} 1330-3153$ & 133033.03 & -315329.1 & 1.14 & unres. \\
\hline J1330-3152 & 133039.44 & -315211.0 & 1.56 & unres. \\
\hline J1330-3105 & 133040.88 & -310547.3 & 3.04 & unres. \\
\hline $\mathrm{J} 1330-3215 \mathrm{~b}$ & 133041.11 & -321521.4 & 0.65 & unres. \\
\hline $\mathrm{J} 1330-3134$ & 133041.21 & -313417.4 & 3.86 & ext. \\
\hline $\mathrm{J} 1330-3141$ & 133042.14 & -314135.7 & 4.18 & unres. \\
\hline J1330-3120 & 133047.27 & -312030.4 & 2.22 & unres. \\
\hline $\mathrm{J} 1330-3143 \mathrm{~b}$ & 133047.95 & -314340.2 & 7.77 & ext. \\
\hline J1330-3148 & 133048.03 & -314845.9 & 1.03 & unres. \\
\hline J1330-3226 & 133048.11 & -322607.5 & 2.61 & unres. \\
\hline J1330-3145 & 133048.27 & -314548.2 & 0.98 & unres. \\
\hline J1330-3146 & 133051.97 & -314600.0 & 1.98 & unres. \\
\hline J1330-3209 & 133052.00 & -320902.3 & 0.73 & unres. \\
\hline J1330-3127 & 133052.33 & -312701.1 & 1.04 & unres. \\
\hline J1330-3204 & 133055.62 & -320401.3 & 9.64 & unres. \\
\hline J1330-3208 & 133059.20 & -320854.2 & 0.51 & unres. \\
\hline J1331-3144 & 133100.60 & -314451.4 & 2.55 & unres. \\
\hline $\mathrm{J} 1331-3140$ & 133101.87 & -314035.3 & 0.60 & unres. \\
\hline J1331-3127 & 133105.38 & -312700.1 & 4.78 & unres. \\
\hline $\mathrm{J} 1331-3154 \mathrm{a}$ & 133106.71 & -315459.9 & 8.48 & ext. \\
\hline J1331-3119 & 133110.88 & -311927.6 & 14.52 & unres. \\
\hline J1331-3139 & 133112.00 & -313927.1 & 9.48 & unres. \\
\hline $\mathrm{J} 1331-3155 \mathrm{a}$ & 133114.14 & -315549.2 & 0.78 & unres. \\
\hline
\end{tabular}


Given the uncertainty in the radio and optical positions, to estimate the reliability of the optical identifications, we adopted the parameter $\mathcal{R}$, defined as:

$\mathcal{R}^{2}=\frac{\Delta_{r-0}^{2}}{\sigma_{0}^{2}+\sigma_{r}^{2}}$

where $\Delta_{r-0}$ is the positional offset between radio and optical coordinates and $\sigma_{0}=1.5 \operatorname{arcsec}$ and $\sigma_{r}=2.5 \operatorname{arcsec}$ are the optical and radio position errors respectively.

We considered as reliable identifications all matches with $\mathcal{R} \leq 3$. Given the extent of the radio emission we found $\mathcal{R}>3$ for a number of sources, but we considered them reliable identifications since the optical counterpart falls within the radio isophotes (further details are given in the Appendix).

The reliability ( $\mathrm{rel}$ ) and completeness (comp) of our sample of identified radio sources was tested following the method suggested by de Ruiter et al. (1977). We note that the reliability represents the fraction of true, i.e. non spurious, optical identifications in the sample, while the completeness provides the fraction of true identifications found with respect to the total number of IDs in the sample. For $\mathcal{R}=3$ we found $\mathrm{rel}=96.3 \%$ and comp $=97.8 \%$; while for $\mathcal{R}=2$ we have rel $=99 \%$ and comp $=68.3 \%$. We note that completeness drops considerably going from $\mathcal{R}=3$ to $\mathcal{R}=2$. For this reason, and given the purpose of the present paper (see Sects. 6.1, 6.3, 7.1 and 7.2), we consider $\mathcal{R}=3$ as the most appropriate choice.

The list of the radio-optical identification is reported in Table 4, where we give:

- Column 1: radio and optical name (as in Yentis et al. 1992), where \# stands for radial velocities from Bardelli et al. (1994, 1998b) and MGP94 from Metcalfe et al. (1994);

- Columns 2 and 3: J2000 radio and optical coordinates;

- Column 4: radio flux density at $1.4 \mathrm{GHz}$ and $b_{\mathrm{J}}$ magnitude;

- Column 5: radio morphology and optical type, where $E=$ elliptical, $\mathrm{S}=$ spiral, IS = interacting system, qso = quasar candidate;

- Column 6: radio power at $1.4 \mathrm{GHz}$ and radial velocity;

- Column 7: $\mathcal{R}$ parameter and $(B-R)$ colours for the optical counterparts in the Shapley Concentration taken from Metcalfe et al. (1994).

The morphological classification of the optical objects given in Table 4 was done by inspection of the DSS- 2 images. The redshift information is taken from Bardelli et al. (1994, 1998b) and Metcalfe et al. (1994).

We found 68 identifications, corresponding to $\sim 40 \%$ of our radio source sample. Among these, 33 (48\% of the identified sources) are located at the redshift of the Shapley Concentration (velocity range $\sim 11000-17500 \mathrm{~km} \mathrm{~s}^{-1}$, Bardelli et al. 1998b).

\section{The radio emission in A3558-C}

\subsection{General comments on the radio galaxies}

The sample of 33 optically identified radio sources in A3558-C includes 18 early-type galaxies and 14 spirals. The optical morphology of J1333-3124 is unavailable (see Table 4).
In Figs. 2 and 3 we show the $1.4 \mathrm{GHz}$ radio contours of the late- and early-type radio galaxies, overlaid on the DSS-2 optical frame. The radio galaxy J1332-3146a is shown separately in Fig. 5; the radio halo at the centre of A3562, including the radio galaxy $\mathrm{J} 1333-3141$ is given in Fig. 4; J1333-3124 is included among the early-type galaxies (Fig. 3).

In Fig. 6 we show the location of the A3558-C earlyand late-type radio galaxies listed in Table 4, overlaid on the $1.4 \mathrm{GHz}$ radio contours of the same region. No difference in the bidimensional distribution of these two classes is obvious from the figure.

In a search for possible segregation effects in the location of the 33 radio galaxies detected in this survey, we examined the velocity distribution of the optical counterparts, and compared it to that of all optical galaxies belonging to the A3558-C region covered in our survey. The optical catalogue was extracted from the sample in Bardelli et al. (1994, 1998b). The radio galaxies appear to be uniformly distributed over the Shapley supercluster velocity range, with a peak of nine objects at $\sim 13000 \mathrm{~km} \mathrm{~s}^{-1}$ (Fig. 7). Six of them belong to the SC 1329-313 group and in particular to the T520 subclump (Bardelli et al. 1998a).

\subsection{The diffuse radio galaxy J1332-3146a}

A remarkable feature of the radio emission in A3558-C is the very low brightness extended tail associated with the radio galaxy J1332-3146a. In Fig. 4 the radio contours of the A3558-C region between the radio halo at the centre of A3562 and J1332-3146a are superposed on the DSS-2 optical frame.

J1332-3146a is identified with the cluster galaxy \#11744 $\left(b_{\mathrm{J}}=14.96\right.$ and velocity $v=13107 \mathrm{~km} \mathrm{~s}^{-1}$, see Table 4), the brightest galaxy in the group SC 1329-313. The projected angular size of the radio emission is $\sim 6^{\prime} \times 4^{\prime}$, corresponding to $\sim 240 \times 160 \mathrm{kpc}$. The radio power is $\log P_{1.4 \mathrm{GHz}}\left(\mathrm{W} \mathrm{Hz}^{-1}\right)=$ 22.52 and its surface brightness is $\sim 0.16 \mathrm{mJy} \mathrm{b}^{-1}$.

We note that the X-ray emission in this region is elongated in the direction of A3562, and compressed towards SC 1327-312, and J1332-3146a is located at the border of the X-ray emission of the SC 1329 group, as is clear from Fig. 5.

The presence of extended emission associated with J1332-3146a was suggested by inspection of $843 \mathrm{MHz}$ Molonglo Observatory Synthes Telescope (MOST) observations and of the NVSS $1.4 \mathrm{GHz}$ image, where the source has a projected angular size of $\sim 4^{\prime} \times 2^{\prime}(\mathrm{V} 2000)$. The overall morphology in our image (Fig. 4) is in very good agreement with the NVSS image.

J1332-3146a was detected as point-like with the ATCA both at $22 \mathrm{~cm}$ and $13 \mathrm{~cm}$, at the resolution of a few arcseconds, and the derived spectral index is $\alpha_{13}^{22}=0.81$ (V2000). We note that the source was labelled J1332-3146 in V2000. The compact morphology and steep spectrum at high resolution seem to rule out any connection between the low brightness extended emission and an active nucleus. In other words, there is no indication that the extended emission imaged with the observations presented here is a tail of a head-tail or wide-angle tail radio source. 
Table 4. Optical identifications.

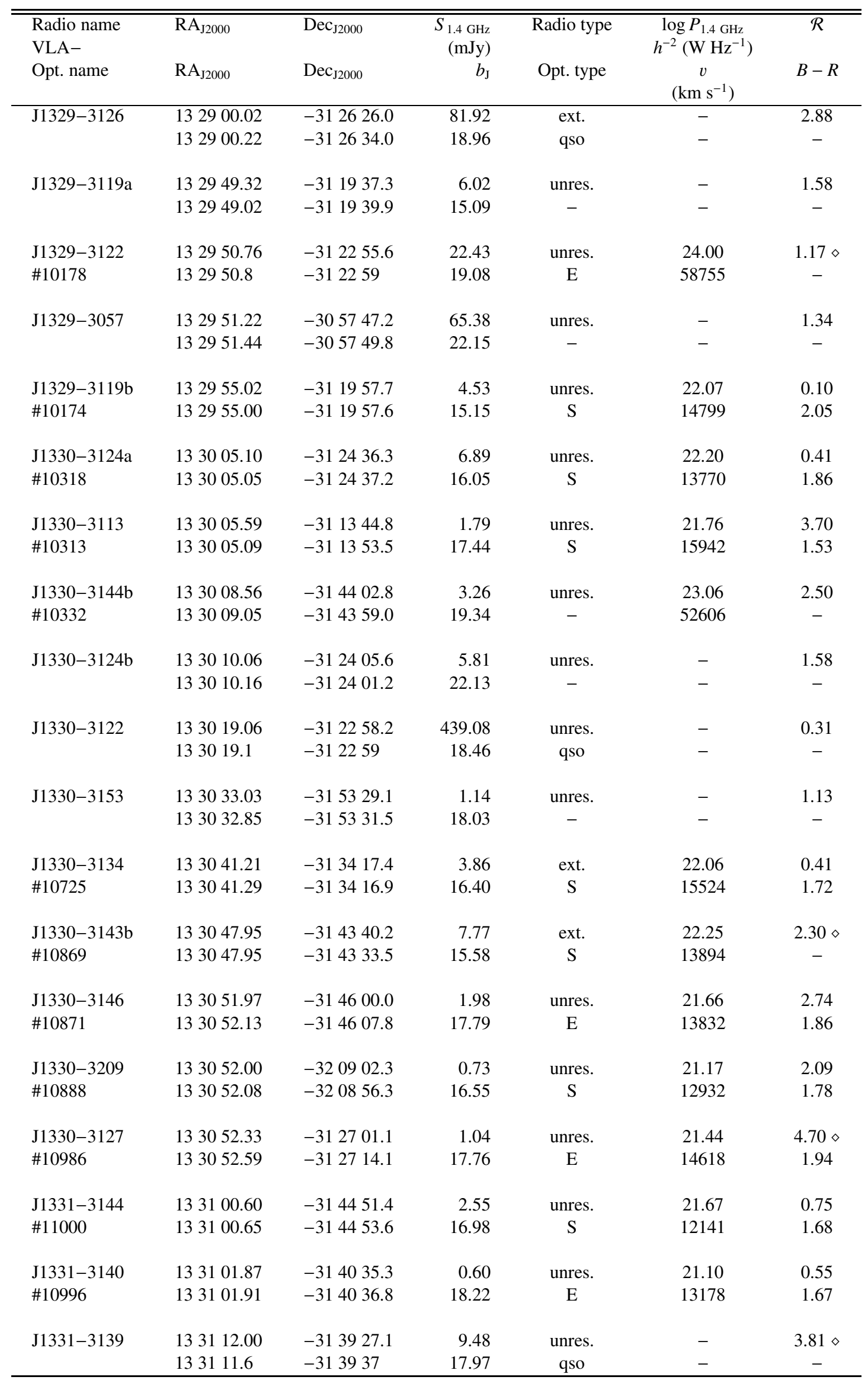



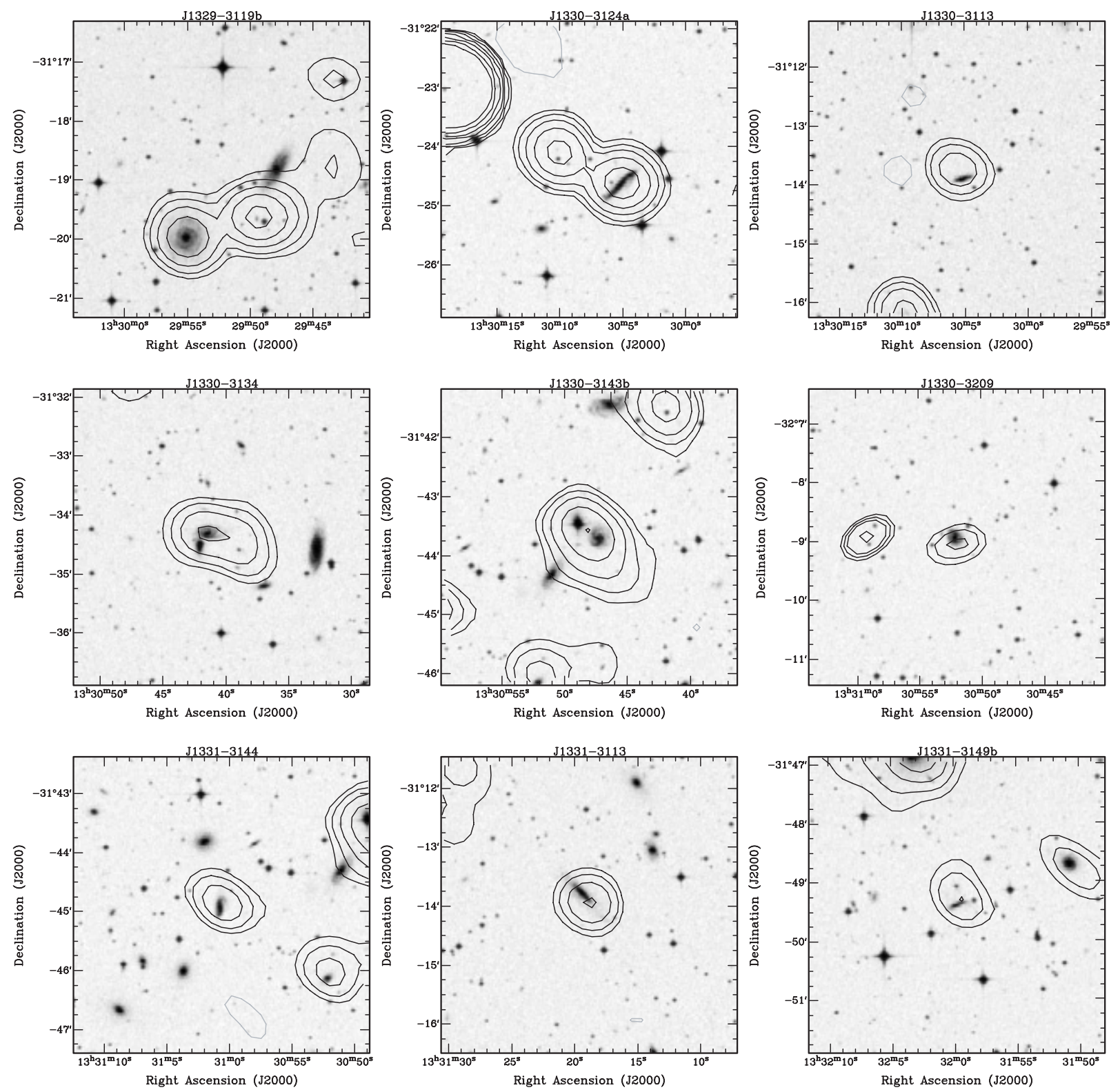

Fig. 2. 1.4 GHz radio contours of the 14 late-type radio galaxies in A3558-C overlaid on the DSS-2 optical frame. Radio contours are as follows. For J1330-3209: $-0.25,0.25,0.30,0.35,0.50,1.00,2.00 \mathrm{mJy} \mathrm{b}^{-1}$; for J1332-3152a: $-0.25,0.25,0.30,0.50,1.00,2.00 \mathrm{mJy} \mathrm{b}^{-1}$; for $\mathrm{J} 1335-3133:-0.50,0.50,0.75,1.00,1.50,2.00 \mathrm{mJy}^{-1}$; for all remaining radio galaxies: $-0.25,0.250 .50,1.00,2.00 \mathrm{mJy}^{-1}$; the restoring beam is $41.98^{\prime \prime} \times 35.13^{\prime \prime}$, PA $55.7^{\circ}$.

Inspection of the radio emission shown in Fig. 4 suggests some important considerations. In particular, $(a)$ the extension of J1332-3146a points towards the radio halo at the centre of A3562; $(b)$ the radio halo is characterised by a filament pointing South-West, towards J1332-3146a; (c) positive residuals of radio emission are clearly visible in the region between the radio halo and J1332-3146a. Excluding the strongest point sources (with flux density $S>5 \sigma$ ), these residuals account for several mJy.

It is possible that the extended emission in J1332-3146a is:

(i) part of a very low brightness cluster scale bridge of emission, extending from the centre of A3562 to the smaller group SC 1329-313, undetectable with the radio instrumentation presently available. This scenario would be similar to the case of the Coma cluster of galaxies, where a bridge of low brightness emission was detected between the central region of the Coma cluster and the merging group NGC 4839 (Giovannini et al. 1993);

ii) the result of reacceleration processes and can therefore be interpreted as revived radio emission. J1332-3146a lies in a region where a merger shock is expected, on the basis of numerical simulations and of the properties of the X-ray emission. 

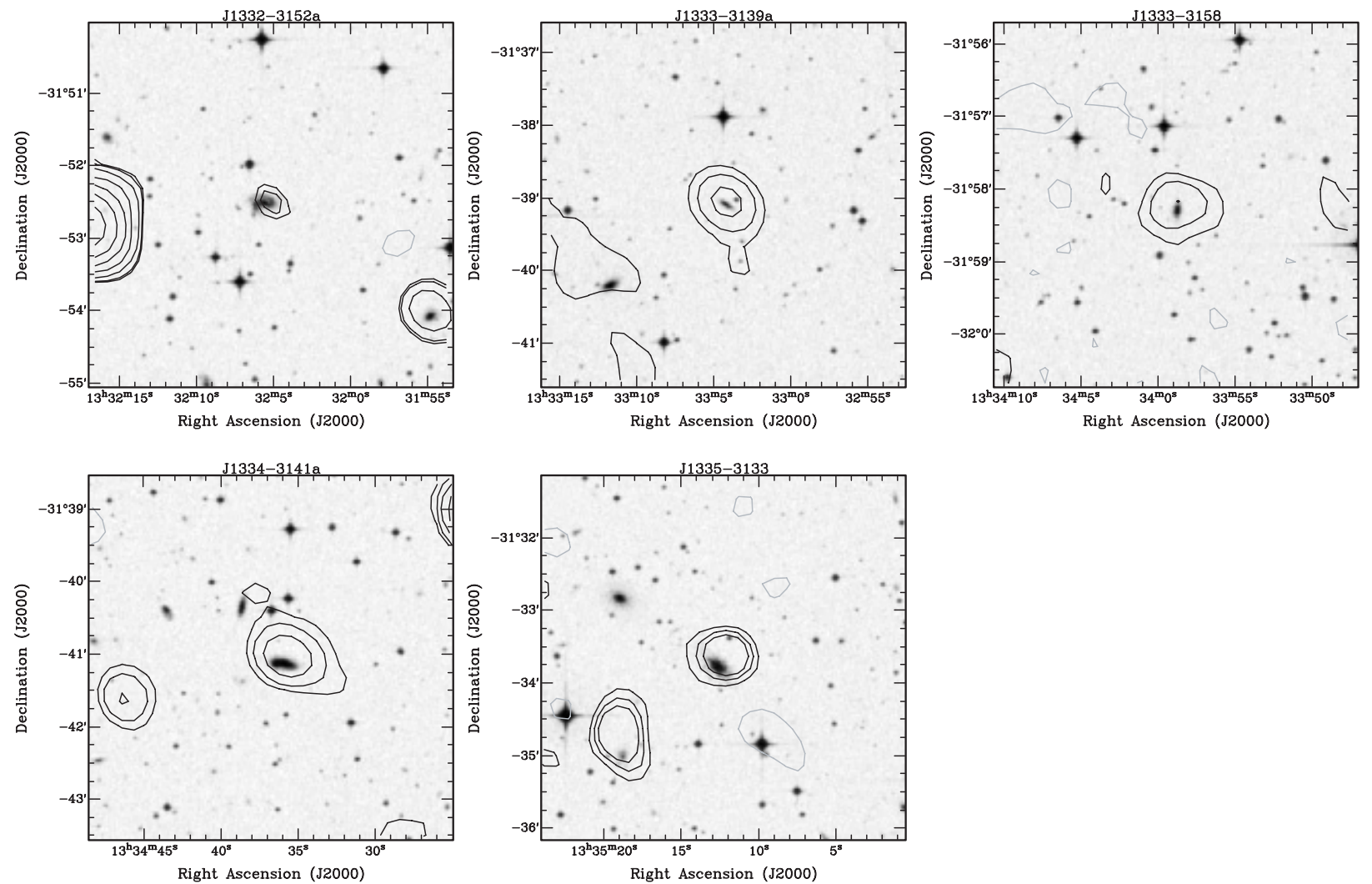

Fig. 2. continued.

\subsection{The population of faint radio galaxies in A3558-C. Enhanced radio activity due to a cluster merger?}

An indicator of starburst activity is the presence of radio emission at power levels of the order $P_{1.4 \mathrm{GHz}} \lesssim 10^{23} h^{-2} \mathrm{~W} \mathrm{~Hz}^{-1}$ (see Condon et al. 2002 for a recent discussion). The majority of the A3558-C radio galaxies in our list has radio power below this threshold. In particular, 12 out of 18 radio emitting ellipticals have $\log P_{1.4 \mathrm{GHz}}\left(\mathrm{W} \mathrm{Hz}^{-1}\right)<21.78$, the lower limit of the radio luminosity function for AGNs derived by LO96 (scaled to the cosmology adopted in our paper) discussed in Sect. 7.1. Among the spiral galaxies with associated radio emission, they all have $\log P_{1.4 \mathrm{GHz}}\left(\mathrm{W} \mathrm{Hz} \mathrm{Hz}^{-1}\right) \leq 22.25$. Including the radio galaxy J1333-3124, whose optical counterpart has no morphological classification, the total number of candidate starburst radio galaxies in our sample is therefore 26.

A detailed optical study of the spectral properties of the galaxies in A3558-C shows that the region between A3562 and the SC groups is populated by a very large fraction of blue galaxies (Bardelli et al. 1998b). To quantify this effect we considered the $(B-R)$ colours of the photometric sample of optical galaxies in this region of Metcalfe et al. (1994): from a $b_{\mathrm{J}}$ vs. $(B-R)$ plot we found that the red sequence is at $(B-R) \sim 2$ and we set $(B-R)<1.7$ as the limit to define a blue object. We cross-correlated our sample of faint radio galaxies with the sample of Metcalfe et al. (1994) and found that 4 spirals and 7 ellipticals are blue. Beyond that, we note that the $(B-R)$ values for all the radio galaxies in A3558-C are lower than the average value for $\mathrm{A} 3558-\mathrm{C}[\langle(B-R)\rangle \sim 2]$, with few very extreme cases (see Table 4). Thus, for half of the faint radio sources in the region under study, indication of starburst radio emission is supported by photometric information. Assuming that there is no contribution from an active nucleus, the star formation rates $(S F R)$ deduced on the basis of the radio emission are in the range $S F R \sim 1.2 \div 7.1 M_{\odot} \mathrm{yr}^{-1}$. This estimate was made using the empirical relation between $S F R$ and radio luminosity in Yun et al. (2001), after scaling our radio luminosity to the cosmology used in their paper.

We compared our results on A3558-C with those obtained by MO03 for A2255, whose radio emission was interpreted in terms of increased radio AGN activity and star formation as consequence of the cluster merger.

For a proper comparison we needed to apply some transformations and corrections to our data. In particular:

(i) for our COSMOS $b_{\mathrm{J}}$ magnitudes, we considered a correction according to Lumsden et al. (1997) for a "saturation" effect which occurs for bright objects due to the lack of dynamic range within the measuring machine; then we assumed $b_{\mathrm{J}} \simeq m_{B}$ (as found by Bardelli et al. 2000) and applied a correction for the galactic absorption of $A_{B}=0.24$ (Schlegel et al. 1998). We converted our magnitudes to the $m_{R_{\mathrm{c}}}$ magnitudes using the $\left(B-R_{\mathrm{c}}\right)$ colours in Fukugita et al. (1995): we adopted $\left(B-R_{\mathrm{c}}\right)=1.48$ for early-type galaxies (average value between $\mathrm{E}$ and $\mathrm{S} 0)$ and $\left(B-R_{\mathrm{c}}\right)=1.00$ for late-type galaxies (average value between spirals and Irr). Finally we computed the $M_{R}$ magnitudes for our sample adopting the MO03 cosmology. 

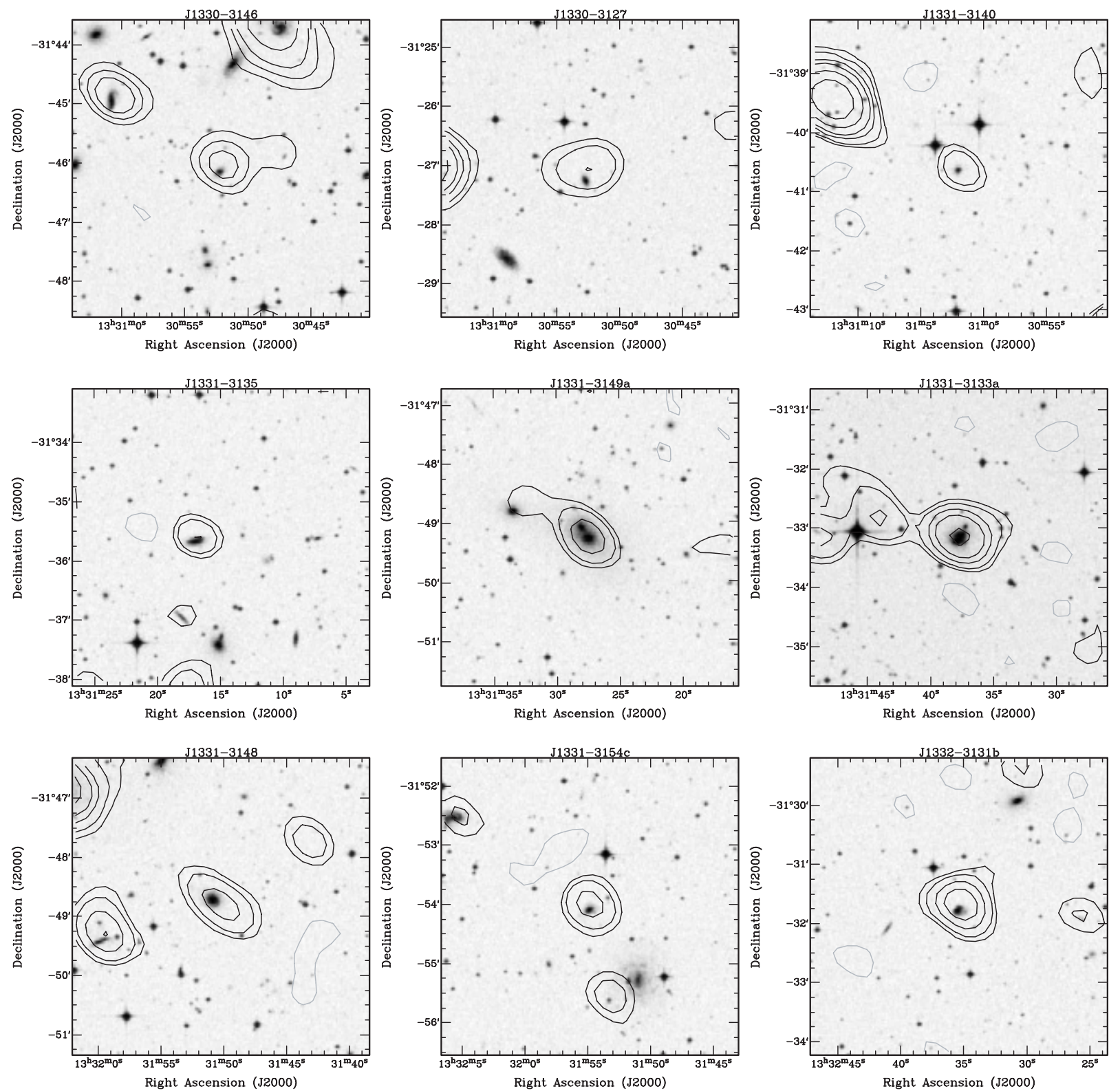

Fig. 3. $1.4 \mathrm{GHz}$ radio contours of the early-type radio galaxies in A3558-C overlaid on the DSS-2 optical frame. Radio contours are: -0.175 , $0.175,0.3,0.6,1,2,4,8,16$ etc. $\mathrm{mJy} \mathrm{b}^{-1}$. For $\mathrm{J} 1330-3146$ and $\mathrm{J} 1334-3126 \mathrm{~b}$ the lowest contour is $-0.3,0.3 \mathrm{mJy}^{-1}$. The restoring beam is $41.98^{\prime \prime} \times 35.13^{\prime \prime}$, PA $55.7^{\circ}$.

(ii) We rescaled the radio powers of our A3558-C radio galaxies to the MO03 cosmology, and selected only those sources with $\log P_{1.4 \mathrm{GHz}}\left(\mathrm{W} \mathrm{Hz}^{-1}\right) \geq 21.84$.

(iii) We extracted a normalization sample from the COSMOS catalogue containing all galaxies within the MO03 limit $M_{R} \leq-20$ in the region of A3558-C covered by our observations. We applied the same magnitude corrections described in point (i). In this case we adopted an average $\left(B-R_{\mathrm{c}}\right)=1.36$ with the assumption of a morphological mix of $73 \%$ ellipticals and $27 \%$ spirals in this region. From the number counts of this sample we subtracted the contribution of field galaxies determined from the ESP survey (Vettolani et al. 1997) which was obtained from the COSMOS catalogue. Also for these objects we considered the same magnitude corrections as in point (i) with $A_{B}=0$ (the ESP survey is at the galactic pole) and $\left(B-R_{\mathrm{c}}\right)=1.15$ which corresponds to a morphological mix for the field of $30 \%$ early-type and $70 \%$ late-type galaxies.

Following MO03 we divided our optical sample in three magnitude bins, i.e. "faint" $\left(-21<M_{R} \leq-20\right)$, "intermediate" $\left(-22<M_{R} \leq-21\right)$ and "bright" $\left(M_{R} \leq-22\right)$, and computed the fraction $\frac{N_{\mathrm{rg}}}{N_{\mathrm{gal}}}$ in each luminosity bin, including all optical morphological types. As in their paper, $N_{\text {gal }}$ and $N_{\text {rg }}$ are respectively the total number of optical galaxies and the total number of radio galaxies in each bin. The results of our analysis are given in Table 5 where we also report the numbers for A2255 and the mean and the dispersion for all the other clusters considered by $\mathrm{MO} 03$ in the $2 \mathrm{Mpc}$ case. The fractions and errors 

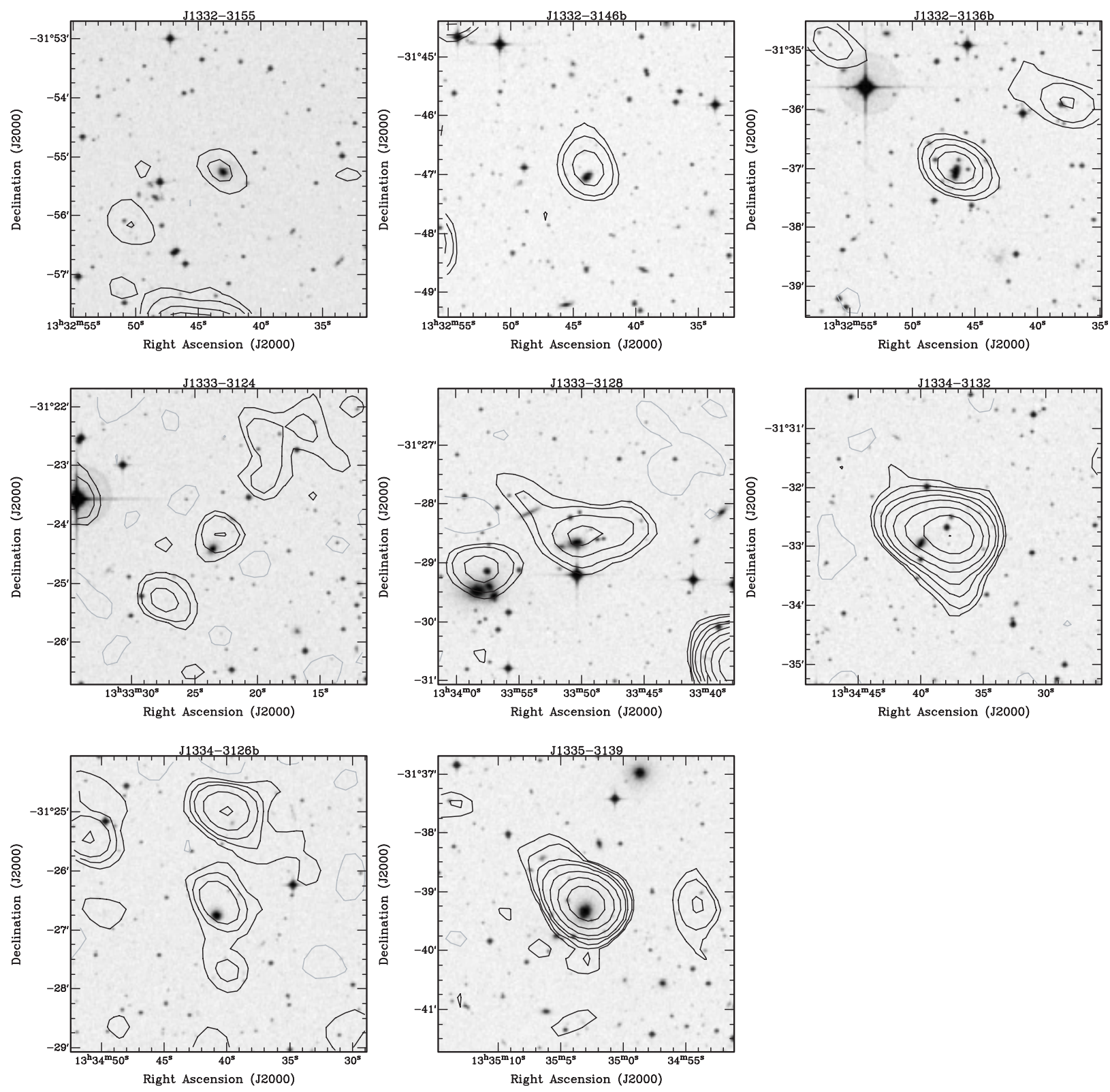

Fig. 3. continued.

associated with the MO03 clusters were computed by us using the numbers given in their paper.

Not surprisingly, the ratio $\frac{N_{\mathrm{rg}}}{N_{\mathrm{gal}}}$ increases going from the faint to the bright optical bin both in A3558-C and in A2255. If we take into account the associated errors, there is only marginal evidence of enhanced fraction of radio emitting galaxies in A3558-C and in A2255 compared to the other clusters considered in MO03.

\section{Statistical analysis of A3558-C: Radio/optical properties}

The radio luminosity function (RLF) is a powerful statistical tool to investigate the radio properties of a galaxy population. To understand if the ongoing major merger in A3558-C has a significant effect on the radio emission of the cluster galaxy population, it is important to compare the radio luminosity function (RLF) for galaxies in this merging environment with the mean RLF for galaxies in normal clusters and in the field.

With this aim we computed the RLF for the radio galaxies in the A3558-C and compared our results with those obtained by LO96 and by Gavazzi \& Boselli (1999, hereafter GB99) respectively for early- and late-type galaxies.

For the comparison we adopted the cosmology used by these authors and scaled the radio powers of our sample. We corrected our $b_{\mathrm{J}}$ magnitudes as described in point (i) of Sect. 6.3.

\subsection{The radio luminosity function of $A G N s$}

We computed the number of radio galaxies expected in the A3558-C region on the basis of the "universal" RLF derived 


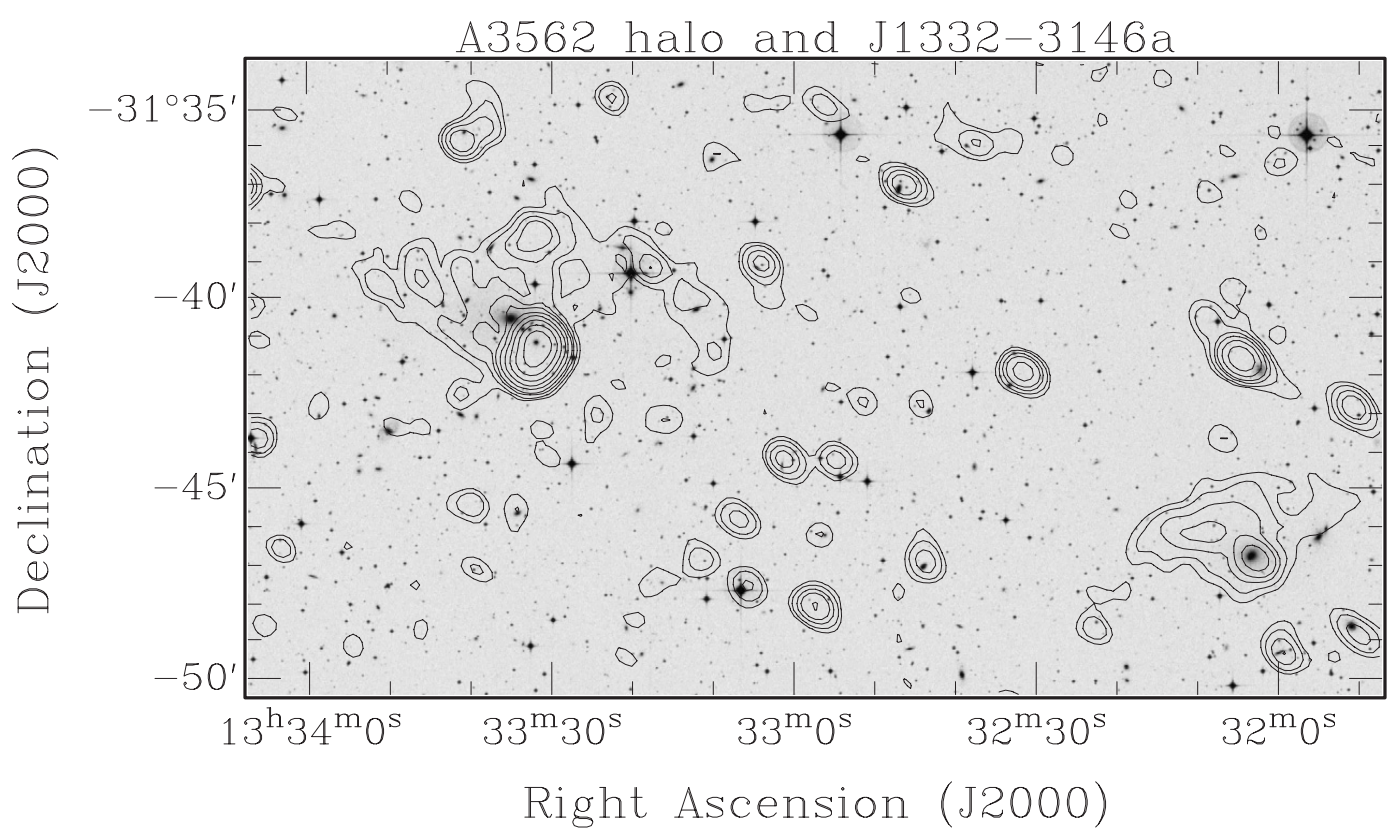

Fig. 4. 1.4 GHz radio contours of the A3558-C region between the A3562 central radio halo and the extended radio galaxy J1332-3146a, overlaid on the optical DSS-2 red frame. Contours are $-0.15,0.15,0.3,0.6,1,2,4,8,16,32,64,128,250 \mathrm{mJy} \mathrm{b}^{-1}$. The restoring beam in the image is $41.98^{\prime \prime} \times 35.13^{\prime \prime}$, in PA $55.7^{\circ}$.

Table 5. Radio-optical galaxy counts.

\begin{tabular}{l|rrr|rr}
\hline \hline Mag interval & $N_{\mathrm{rg}}$ & \multicolumn{2}{c|}{$\begin{array}{l}N_{\mathrm{gal}} \\
(\mathrm{A} 3558-\mathrm{C})\end{array}$} & $\begin{array}{r}\% \\
(\mathrm{~A} 2255)\end{array}$ & $\begin{array}{r}\% \\
\text { Other clusters }\end{array}$ \\
\hline$-21<M_{R} \leq-20$ & 3 & 88.4 & $3.4 \% \pm 2.0$ & $7.2 \% \pm 2.7$ & $1.6 \% \pm 1.4$ \\
$-22<M_{R} \leq-21$ & 9 & 37.8 & $23.8 \% \pm 7.9$ & $15.3 \% \pm 4.8$ & $20.3 \% \pm 12.8$ \\
$M_{R} \leq-22$ & 6 & 13.7 & $43.8 \% \pm 17.9$ & $56.5 \% \pm 21.3$ & $23.3 \% \pm 23.1$ \\
\hline
\end{tabular}

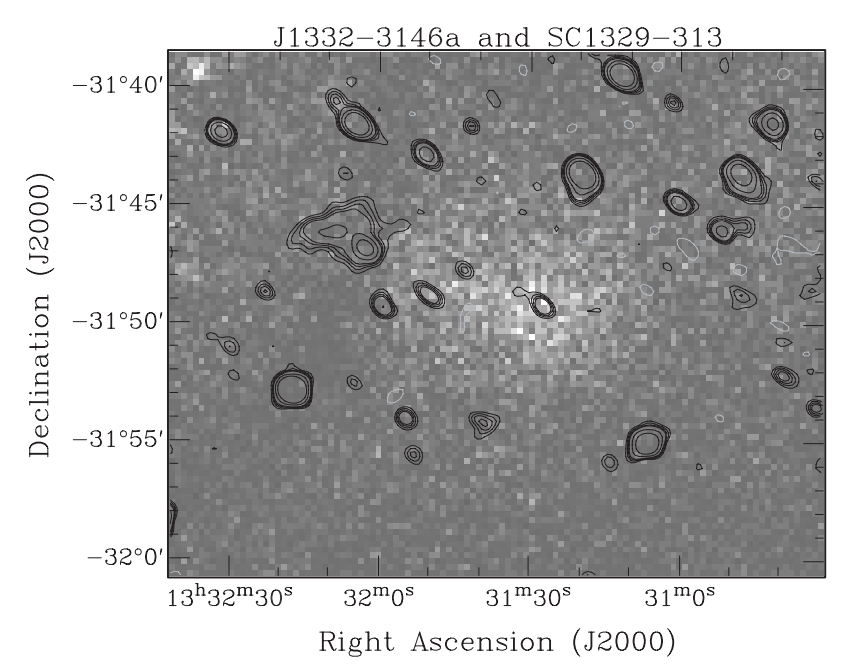

Fig. 5. 1.4 GHz VLA radio emission from the group SC 1329-313 (grey scale) superposed to the ROSAT X-ray emission. The extended radio galaxy $\mathrm{J} 1332-3146 \mathrm{a}$ is located just outside the X-ray peak, in the North-East direction. The $F W H M$ of the restoring beam of the radio image is $41.98^{\prime \prime} \times 35.13^{\prime \prime}$, in PA $55.7^{\circ}$. The radio contours are -0.2 , $0.2,0.3,0.4,0.5,1,2 \mathrm{mJy} \mathrm{b}^{-1}$.

by LO96 and compared it to the number of objects actually detected in our survey.
The analysis carried out by LO96 includes radio galaxies with $\log P_{1.4 \mathrm{GHz}}\left(\mathrm{W} \mathrm{Hz}{ }^{-1}\right) \geq 22.03$ and optical counterparts brighter than $M_{R}=-20.5$. This magnitude limit corresponds to $m_{R}=16.05$ at the distance of the Shapley supercluster.

We estimated that the total number of early-type galaxies in the A3558-C region located at the distance of the Shapley Concentration is 65 (see V2000 for further details). On the basis of LO96, the fraction of early-type galaxies with $M_{R} \leq-20.5$ expected to be radio loud at a power level $\left.\log P_{1.4 \mathrm{GHz}}(\mathrm{W} \mathrm{Hz})^{-1}\right) \geq 22.03$ is $6(\sim 9.2 \%)$, in good agreement with the seven radio galaxies in our sample matching the LO96 constraints.

Conversely, V2000 found that the RLF for early-type galaxies of the whole A3558 complex, including the cluster A3558 and the westernmost A3556, is significantly lower than the "universal" RLF by LO96. These results suggest that the effect of cluster mergers on the AGN-type radio emission may be a very complex phenomenon, possibly depending on the stage of the merger. This issue will be further discussed in Sect. 8 .

\subsection{The radio luminosity function of late-type galaxies}

The role of environment on the radio emission of spiral galaxies has been investigated in a number of papers. 


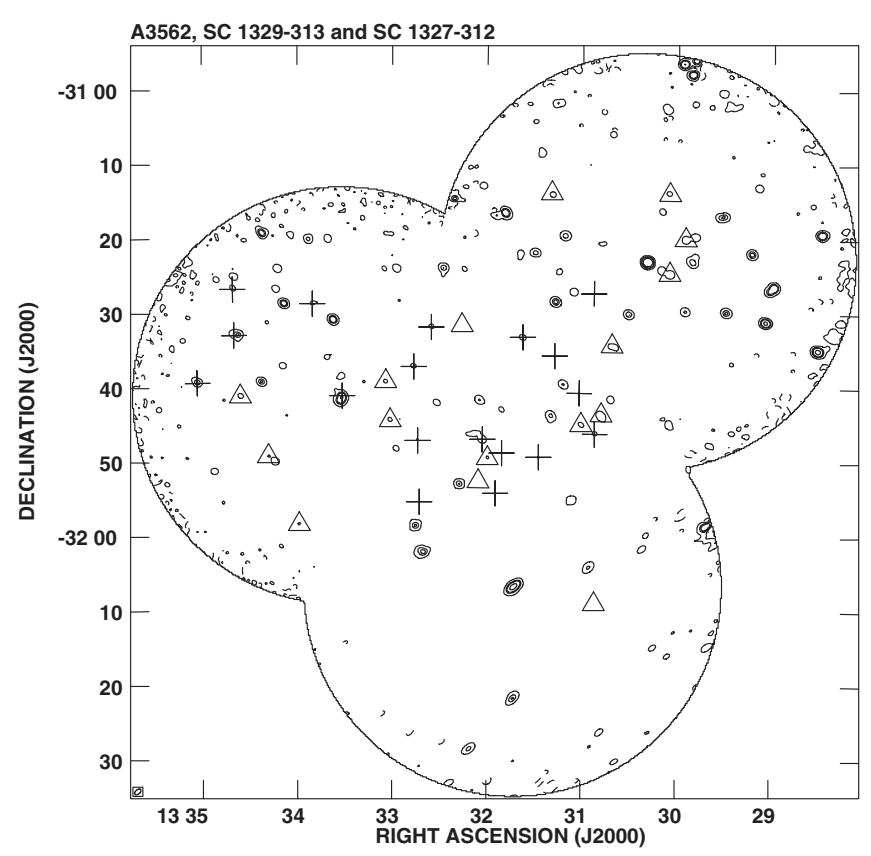

Fig. 6. Location of the Shapley Concentration optical counterparts overlaid on the $1.4 \mathrm{GHz}$ radio contours of the A3562 and SC groups region. Triangles represent late-type galaxies and crosses stand for early-type galaxies. The radio contours are $-0.85,0.85,6.80,13.60$ and $54.40 \mathrm{mJy} \mathrm{b}^{-1}$. The galaxy without optical classification was included among the early-type galaxies.

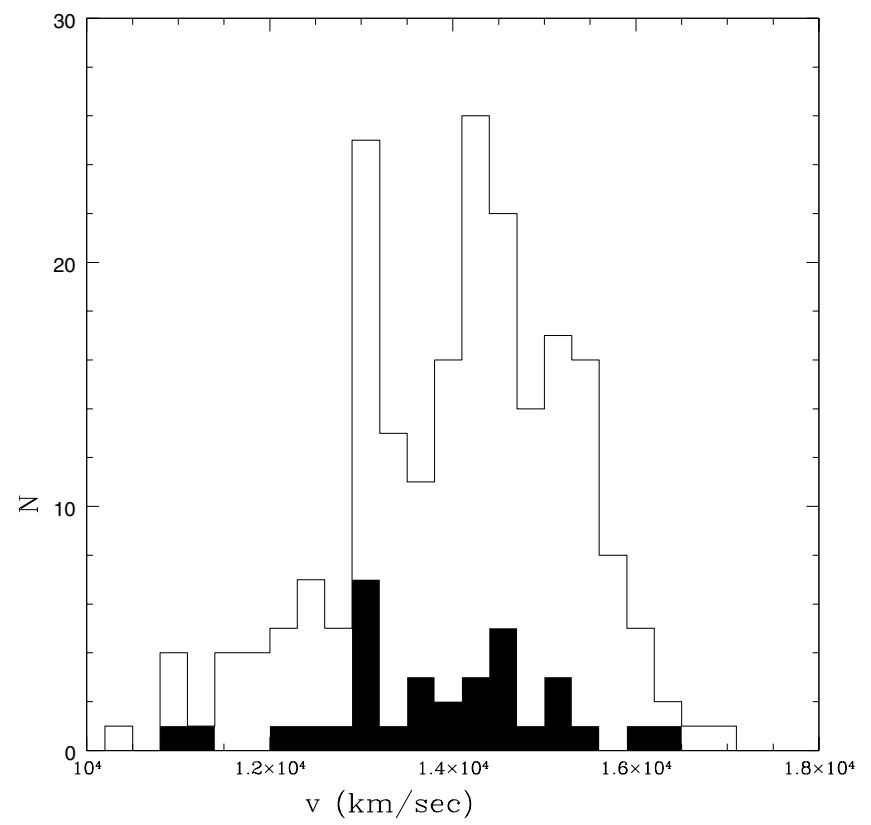

Fig. 7. Histogram of the velocity distribution of the radio galaxies in the A3562 and SC groups region given in Table 4 (black bins), compared to the velocity distribution of all galaxies in the Shapley Concentration. The width of each velocity bin is $300 \mathrm{~km} \mathrm{~s}^{-1}$.

Jaffe \& Perola (1976) found that radio emitting spirals in the Coma cluster have a radio excess compared to field galaxies. Gavazzi \& Jaffe (1986) confirmed these results by comparing the RLF of late-type galaxies within and outside rich clusters. GB99 computed the RLF of spiral galaxies, in terms of radio-optical flux density ratio, in nearby different environments (rich and poor clusters, and the field), and found that late-type galaxies in rich clusters develop radio sources more frequently than galaxies in poor clusters and in the field. They also found a correlation between the radio excess and the velocity deviations with respect to the cluster average velocity. GB99 suggested that these results are coherent with a ram pressure scenario: galaxies in fast motion through the intracluster medium experience enough dynamical pressure to compress their magnetic field on the up-stream side, form a tail-like radio structure on the down-stream side and produce a net enhancement of the radio continuum activity.

We addressed the question of whether the late-type galaxy population in the merging environment of the A3558-C region behaves like those in rich clusters, or if it shows even more enhanced radio excess. To this aim we compared the RLF of late-type galaxies in this region with the results obtained by GB99. We extracted a subsample of 209 objects from the GB99 sample, with Zwicky magnitude $m_{z}<15.7$ and flux density $S_{1.4 \mathrm{GHz}} \geq 2.25 \mathrm{mJy}$, corresponding to the $5 \sigma$ level of the NVSS survey (radio data in GB99 are taken mostly from this survey). This flux density limit implies a radio power limit of $\log P_{1.4 \mathrm{GHz}}\left(\mathrm{W} \mathrm{Hz}{ }^{-1}\right)=20.74$ at the distance of the Cancer cluster, the nearest in their sample $(z=0.015)$.

For a proper comparison, we computed the $b_{\mathrm{J}}$ magnitude limit corresponding to the $m_{z}$ limit in GB99. First we converted $m_{z}$ in $m_{B}$ according to the relationship $m_{B}=m_{z}-0.35$ (Gaztañaga \& Dalton 2000), assuming $b_{\mathrm{J}} \simeq m_{B}$ (Sect. 6.3). Then we applied a distance modulus of $1.5 \mathrm{mag}$ and a $0.24 \mathrm{mag}$ correction for the galactic absorption (Schlegel et al. 1998). We obtained a limit of $b_{\mathrm{J}}^{\prime}=17.09$, where $b_{\mathrm{J}}^{\prime}$ is the magnitude corrected as described above.

For each late-type radio galaxy in A3558-C we applied a correction for the internal extintion. This latter was determined according to Gavazzi \& Boselli (1996) using an average value of 0.60 for the $D_{B}$ (type) coefficient, which corresponds to $\mathrm{Sb}$ galaxies in the $B$ band.

We selected, at the same radio power limit as GB99, a sample of spiral galaxies in A3558-C with $b_{\mathrm{J}}^{\prime} \leq 17.09$. The resulting sample includes 13 objects and is presented in Table 6. Note that the radio powers in Table 6 are scaled to the cosmology adopted by GB99.

For each radio galaxy of our sample we computed the radio/optical ratio, defined in GB99 as $R_{B}=S_{1.4 \mathrm{GHz}} / k \times 10^{-0.4 \times b_{\mathrm{J}}^{\prime}}$, where $k=4.44 \times 10^{6}$ is the factor appropriate to transform in mJy the $b_{\mathrm{J}}$ magnitude. We note that this quantity is independent of distance. According to Gavazzi et al. (1996) $R_{B}$ gives the ratio of the radio emission per unit light emitted by the relatively young stellar population. We compared the distribution of $R_{B}$ ratios for the late-type galaxy sample in A3558C to the subsample extracted from GB99 (Fig. 8). That the $R_{B}$ distribution of our sample does not change significantly if we use $D_{B}$ (type) coefficients corresponding to morphological types different from $\mathrm{Sb}$.

The limited number of galaxies in A3558-C does not allow us to draw firm conclusions, however some considerations can be made. The most remarkable features in Fig. 8 are the peaked distribution (in the first bin) for the galaxies in poor 

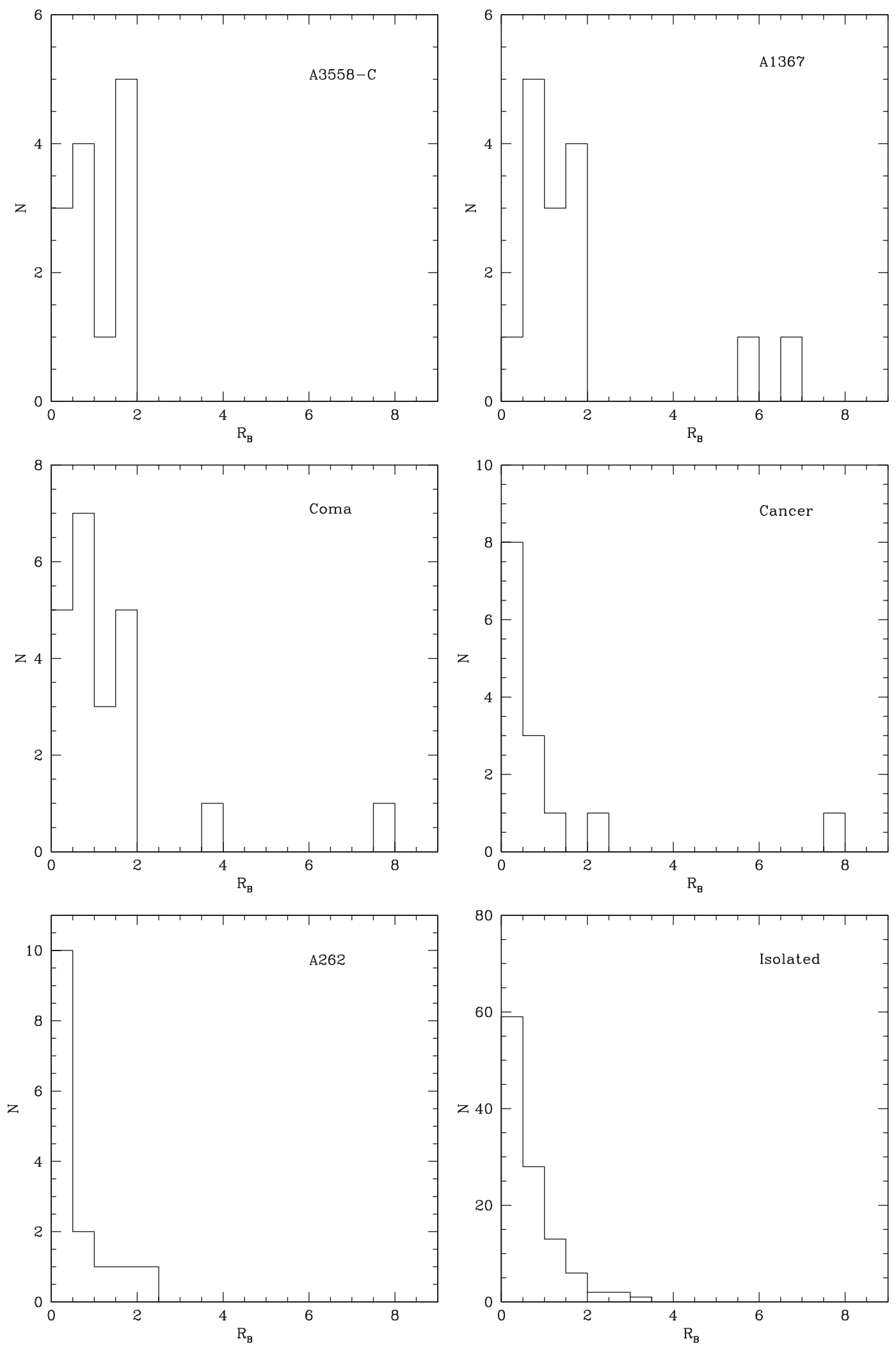

Fig. 8. Distributions of the $R_{B}$ radio/optical ratios for the late-type galaxies in A3558-C, A1367, Coma, Cancer, A262 and for isolated galaxies in the Coma supercluster.

environments, and the presence of galaxies (though a limited number) with a strong excess of radio emission both in rich and poor environments.
Considering Poisson errors in each bin, the distribution of radio late-type galaxies in A3558-C is substantially flat up to $R_{B}=2$, similar to the distributions in Coma and A1367. 
Table 6. Sample of late-type radio galaxies used for the RLF.

\begin{tabular}{lccccc}
\hline \hline $\begin{array}{l}\text { Radio } \\
\text { name }\end{array}$ & $\begin{array}{c}\text { Optical } \\
\text { name }\end{array}$ & $b_{\mathrm{J}}^{\prime}$ & $\begin{array}{c}S_{1.4 \mathrm{GHz}} \\
\mathrm{mJy}\end{array}$ & $\begin{array}{c}\log P_{1.4 \mathrm{GHz}} \\
\mathrm{W} \mathrm{Hz}^{-1}\end{array}$ & $R_{B}$ \\
\hline $\mathrm{J} 1329-3119 \mathrm{~b}$ & $\# 10174$ & 14.39 & 4.53 & 22.07 & 0.58 \\
$\mathrm{~J} 1330-3124 \mathrm{a}$ & $\# 10318$ & 14.52 & 6.89 & 22.20 & 0.99 \\
$\mathrm{~J} 1330-3113$ & $\# 10313$ & 16.42 & 1.79 & 21.76 & 1.49 \\
$\mathrm{~J} 1330-3134$ & $\# 10725$ & 15.66 & 3.86 & 22.06 & 1.60 \\
$\mathrm{~J} 1330-3143 \mathrm{~b}$ & $\# 10869$ & 14.96 & 7.77 & 22.25 & 1.69 \\
$\mathrm{~J} 1330-3209$ & $\# 10888$ & 15.91 & 0.73 & 21.17 & 0.38 \\
$\mathrm{~J} 1331-3144$ & $\# 11000$ & 16.20 & 2.55 & 21.67 & 1.73 \\
$\mathrm{~J} 1331-3113$ & $\# 11243$ & 14.88 & 2.38 & 21.83 & 0.48 \\
$\mathrm{~J} 1331-3149 \mathrm{~b}$ & $\# 11751$ & 16.61 & 1.82 & 21.58 & 1.81 \\
$\mathrm{~J} 1332-3152 \mathrm{a}$ & $\# 11753$ & 15.67 & 0.49 & 21.04 & 0.20 \\
$\mathrm{~J} 1333-3158$ & $\# 12912$ & 17.07 & 1.00 & 21.52 & 1.52 \\
$\mathrm{~J} 1334-3141 \mathrm{a}$ & $\# 13198$ & 15.56 & 2.11 & 21.52 & 0.80 \\
$\mathrm{~J} 1335-3133$ & $\# 13629$ & 15.52 & 2.21 & 21.69 & 0.80 \\
\hline
\end{tabular}

We estimated the similarity between A3558-C and Coma applying a Kolmogoroff-Smirnov (KS) test to the two distributions and found that the probability that they are the same distribution is $\sim 92.4 \%$. A KS test applied to A3558-C and the isolated galaxies gives a probability of only $\sim 3.6 \%$.

The late-type galaxies with $R_{B} \gtrsim 4$ are missing in A3558-C (respectively 2/15 and 2/22 for A1367 and Coma), however this could be due to a lack of statistics, since only 13 spirals in A3558-C are included in the analysis.

The statistical properties of the radio emission from spiral galaxies in A3558-C are similar to those in rich and dynamically evolved environments.

\section{Discussion and conclusions}

In this paper we presented deep 1.4 GHz VLA observations of the major cluster merger in the A3558 complex. The area under study, A3558-C, defined as the region between the centres of the two Abell clusters A3558 and A3562, including also the two groups SC 1329-213 and SC 1327-312, is thought to have recently experienced a major merger between two massive clusters. What we see now is expected to be the result of the first core-core encounter. The observational properties in this region, together with numerical simulations, suggest that A3558 is the main cluster, while the whole chain, beyond A3558, is the remains of the colliding cluster (Bardelli et al. 2002).

A picture is emerging in which radio AGN and starburst activity, radio relics and halos, or the lack thereof, are signatures of cluster mergers at different stages. In particular, Venturi et al. (2002) proposed an evolutionary merger sequence to account for the diversity in the radio properties of the three main merging cluster systems in the core of the Shapley Concentration; Kempner \& Sarazin (2001) postulated that radio halos and relics may form at different times during mergers; MO03 explained the different fraction of radio-emitting galaxies (starburst and AGNs) in a cluster sample as due to different merger stages. With this study we have a unique opportunity to connect the observed properties in the radio band with a well defined cluster merger stage. This is of crucial importance for a better understanding of the complex effects of cluster mergers, with particular emphasis on the stage of the merger.

The most relevant results of our analysis can be summarised as follows:

(i) a faint radio halo is found at the centre of A3562, whose properties are consistent with the idea that it is a young source at the beginning of the reacceleration phase (V2003) induced by a recent merger event;

(ii) the origin of the extended emission in the radio galaxy J1332-3146a, associated with the dominant galaxy in the group SC 1329-313, is unclear. No radio jets are present in the nuclear region of J1332-3146a, and the nuclear radio component has a steep spectrum. It is possible that this extended emission originates in the cluster (see Sect. 6.2), being either (a) a "bright" area of a very low brightness bridge of radio emission, connecting A3562 and SC 1329-313, or (b) a "revived" radio emission region, where pre-existing electrons were reaccelerated;

(iii) we found 33 radio sources associated with A3558-C galaxies, most of them with radio emission at low power levels. Our analysis (see Sect. 6.3) suggests that 26/33 radio galaxies are candidate starbursts. Among them, 11 also show a blue excess;

(iv) the total number of radio AGNs detected in this region is consistent with the expectations from the RLF of LO96, suggesting that the cluster merger has not affected the probability of an early-type galaxy developing a nuclear radio source;

(v) the distribution of the radio/optical ratios for the spirals in A3558-C is similar to what is found in rich and evolved environments.

The main questions are if and how this wealth of observables is connected to the cluster merger in this region. Following from the finding of the radio halo in the centre of A3562 (V2003), 
the radio properties of the Shapley galaxies in this region may contain important pieces of information.

V2000 showed that the RLF for early-type galaxies in the whole A3558-C shows a deficit of radio galaxies compared to the "universal" RLF presented in LO96 (V2000), over the whole power range. On the other hand, the RLF for AGNs presented here matches the expectations of LO96; furthermore the outskirts of the chain (A3556 and A3562) contain the largest fraction of Shapley radio galaxies (see also Sect. 2). This suggests that A3558 itself, the most massive cluster in the chain, is the main cause of the lack of radio sources in the RLF found in V2000. A possible explanation is the key role of A3558 in the merger, i.e. it is experiencing the most dramatic effects of the merger, being the result of a head-on interaction between the cores of the two colliding clusters. If our interpretation is correct, then the role of cluster merger on the radio emission from AGNs may depend on the age, strength and type of impact of the merger.

Optical photometric information on the faint population of radio galaxies in A3558-C confirms that at least 50\% of these objects are most likely starburst candidates. However, a radio/optical analysis carried out following MO03 provides only weak evidence that the fraction of radio-emitting galaxies in A3558-C is higher than in non-merging environments.

The statistical results on the late-type galaxies show that the radio emission in the A3558-C spirals is similar to that in rich and dynamically evolved clusters. This suggests that the radio emission in spiral galaxies may be one of the first "parameters" to react to a cluster merger event.

In V2003 we showed that the radio halo at the centre of A3562 is consistent with a reacceleration phase which started $\sim 10^{8}$ years ago. This ongoing merger is therefore advanced, in the sense that the core-core encounter has already taken place, but it is still young if compared to the total duration expected for a cluster merger, i.e. $10^{9} \mathrm{yr}$.

From our global study of the A3558 complex (V2000, V2003 and the present paper), we can conclude that cluster mergers provide the necessary energy to lead to the formation of radio halos, however their influence on the radio emission from individual cluster galaxies is more uncertain. In particular, our data provide only marginal support for the claim of enhanced starburst emission made by MO03. Moreover, the radio emission of AGN origin remarkably is affected only in the region which has experienced the most violent consequences of the core-core encounter, where we detected a lack of AGN-type radio sources compared to the "universal" luminosity function (V2000). Finally, the statistical properties of the radio emission in spirals in an on-going merger are similar to those in dynamically evolved clusters. This suggests that the radio emission in spirals has already "reacted" to the merger event over a timescale of $\sim 10^{8} \mathrm{yr}$.

Acknowledgements. This work has been partially supported by the Italian Space Agency grants ASI-I-R-105-00, ASI-I-R-037-01 and ASI-I-R-063-02, and by the Italian Ministery (MIUR) grant COFIN2001 "Clusters and groups of galaxies: the interplay between dark and baryonic matter". NRAO is a facility of the National Science Foundation, operated under cooperative agreement by Associated Universities, Inc.
This work has made use of the NASA/IPAC Extragalactic Database NED which is operated by the JPL, California Institute of Technology, under contract with the National Aeronautics and Space Administration.

\section{Appendix A: Notes to optical identifications}

- J1329-3122a: this radio source is the blend of two sources in the ATCA $22 \mathrm{~cm}$ catalogue: the pointlike J1329-3122, associated with a 18.54-mag galaxy, and the FRII J1329-3123, identified with the elliptical cluster galaxy \#10178 $\left(b_{\mathrm{J}}=19.08\right)$. The location of this latter is coincident with the radio emission peak of the 1.4 GHz VLA image, so we consider J1329-3122a associated with the elliptical cluster galaxy;

- J1330-3143b: this source is identified with the spiral \#10869, but other two cluster galaxies fall within the radio isophotes of this extended source.

- J1330-3127: this identification is uncertain since the candidate optical counterpart, which falls within the radio contours, is misplaced with respect to the radio emission peak.

- J1331-3139: this source is the blend of the two J1331-3139a and $\mathrm{J} 1331-3139 \mathrm{~b}$ radio sources of the ATCA-22 cm catalogue. The latter is identified with the 17.97-mag quasar.

- J1331-3143: this source is probably the blend of more sources. A galaxy with $b_{\mathrm{J}}=16.82$ and $z=0.044$ is located within the radio contours, at $\sim 45$ arcsec from the radio emission peak.

- J1331-3155b: a spiral galaxy, with $b_{\mathrm{J}}=15.39$ and $v=$ $4505 \mathrm{~km} \mathrm{~s}^{-1}$ is located at the edge of the radio contours, at $\sim 37$ arcsec from the emission peak.

- J1332-3141a: a cluster galaxy with $b_{\mathrm{J}}=16.96$ and $v=$ $10732 \mathrm{~km} \mathrm{~s}^{-1}$ lies within the radio contours of the source, at $\sim 50$ arcsec from the emission peak.

- J1334-3132: this source is the blend of the three ATCA $22 \mathrm{~cm}$ sources J1334-3132a, J1334-3132b e J1334-3132c. $\mathrm{J} 1334-3132 \mathrm{c}$ is associated with the 17.30-mag elliptical galaxy \#13281. This galaxy falls within the radio contours of the $1.4 \mathrm{GHz}$ VLA image, but it is dislocated with respect to the emission peak.

\section{References}

Abell, G. O., Corwin, H. G., \& Olowin, R. P. 1989, A\&AS, 70, 1 (ACO)

Balogh, M. L., Schade, D., Morris, S. L., et al. 1998, ApJ, 504, L75

Bardelli, S., Zucca, E., Vettolani, G., et al. 1994, MNRAS, 267, 665

Bardelli, S., Zucca, E., Malizia, A., et al. 1996, A\&A, 305, 435

Bardelli, S., Pisani, A., Ramella, M., Zucca, E., \& Zamorani, G. 1998a, MNRAS, 300, 589

Bardelli, S., Zucca, E., Zamorani, G., Vettolani, G., \& Scaramella, R. 1998b, MNRAS, 296, 599

Bardelli, S., Zucca, E., Zamorani, G., Moscardini, L., \& Scaramella, R. 2000, MNRAS, 312, 540

Bardelli, S., De Grandi, S., Ettori, S., et al. 2002, A\&A, 382, 17

Bekki, K. 1999, ApJ, 510, L15

Bothun, G., \& Dressler, A. 1986, ApJ, 301, 57

Breen, J., Raychaudhury, S., Forman, J., \& Jones, C. 1994, ApJ, 424, 59

Burns, J. O., Roettiger, K., Ledlow, M., \& Klypin, A. 1994, ApJ, 427, L87 
Condon, J. J., Cotton, W. D., \& Broderick, J. J. 2002, AJ, 124, 675 de Ruiter, H. R., Willis, A. G., \& Arp, H. C. 1977, A\&AS, 28, 211 Dwarakanath, K. S., \& Owen, F. N. 1999, AJ, 118, 625

Ettori, S., Fabian, A. C., \& White, D. A. 1997, MNRAS, 289, 787

Ettori, S., Bardelli, S., De Grandi, S., et al. 2000, MNRAS, 318, 239

Fujita, Y., Tazikawa, M., Nagashima, M., \& Enoki, M. 1999, PASJ, 51, L1

Fukugita, M., Shimasaku, K., \& Ichikawa, T. 1995, PASP, 107, 945

Gaztañaga, E., \& Dalton, G. B. 2000, MNRAS, 312, 417

Gavazzi, G., \& Jaffe, W. 1986, ApJ, 310, 53

Gavazzi, G., \& Boselli, A. 1996, Astroph. Lett. Commun., 35, 1

Gavazzi, G., \& Boselli, A. 1999, A\&A, 343, 93 (GB99)

Giovannini, G., Feretti, L., Venturi, T., Kim, K.-T., \& Kronberg, P. P. 1993, ApJ, 406, 399

Gunn, J. E., \& Gott, J. R. 1972, ApJ, 176, 1

Hambly, N. C., MacGillivray, H. T., Read, M. A., et al. 2001, MNRAS, 326,1279

Jaffe, W., \& Perola, G. C. 1976, A\&A, 46, 275

Kempner, J. C, \& Sarazin, C. L. 2001, ApJ, 548, 639

Ledlow, M. J., \& Owen, F. N. 1996, ApJ, 112, 9 (LO96)

Lumsden, S. L., Collins, C. A., Nichol, R. C., Eke, V. R., \& Guzzo, L. 1997, MNRAS, 290, 119

Maddox, S. J., Efstathiou, G., Sutherland, W. J., \& Loveday, J. 1990, MNRAS, 243, 692

Metcalfe, N., Godwin, J. G., \& Peach, J. V. 1994, MNRAS, 267, 431

Miller, N. A., Owen, F. N., \& Hill, J. M. 2003, AJ, 125, 2393

Miller, N. A., \& Owen, F. N. 2003, AJ, 125, 2427 (MO03)
Owen, F. M., Ledlow, M. J., Keel, W. C., \& Morrison, G. E. 1999, AJ, 118,633

Prandoni, I., Gregorini, L., Parma, P., et al. 2000, A\&AS, 146, 41

Prandoni, I., Gregorini, L., Parma, P., et al. 2001, A\&A, 365, 392

Roettiger, K., Loken, C., \& Burns, J. O. 1997, ApJS, 109, 307

Schlegel, D. J., Finkbeiner, D. P., \& Davis, M. 1998, ApJ, 500, 525

Unewisse, A. M., Hunstead, D. W., \& Pietrzynski, B. 1993, Publ. Astron. Soc. Austr., 10, 229

Venturi, T., Bardelli, S., Morganti, R., \& Hunstead, R. W. 1997, MNRAS, 285, 898

Venturi, T., Bardelli, S., Morganti, R., \& Hunstead, R. W. 1998, MNRAS, 298, 1113

Venturi, T., Bardelli, S., Morganti, R., \& Hunstead, R. W. 2000, MNRAS, 314, 594 (V2000)

Venturi, T., Bardelli, S., Zambelli, G., Morganti, R., \& Hunstead, R. W. 2001, MNRAS, 324, 1131

Venturi, T., Bardelli, S., Zagaria, M., Prandoni, I., \& Morganti, R. 2002, A\&A, 385, 39

Venturi, T., Bardelli, S., Dallacasa, D., et al. 2003, A\&A, 402, 913 (V2003)

Vettolani, G., Zucca, E., Zamorani, G., et al. 1997, A\&A, 325, 954

Vollmer, B., Braine, J., Balkowski, C., Cayatte, V., \& Duschl, W. J. 2001, A\&A, 374, 824

Yentis, D. J., Cruddace, R. G., Gursky, H., et al. 1992, in Digitised Optical Sky Surveys, ed. H. T. MacGillivray, \& E. B. Thomson (Kluwer Academic Publishers), 67

Yun, M. S., Reddy, N. A., \& Condon, J. J. 2001, ApJ, 554, 803 